\title{
Propiedades mecánicas de la aleación AZ31 procesada por una ruta eco-sostenible ${ }^{(\cdot)}$
}

\author{
F. D’Errico*, G. Garcés**, M. Hofer***, S.K. Kim****, P. Pérez**, S. Cabeza** y P. Adeva**
}

\begin{abstract}
Resumen Recientemente se ha demostrado la posibilidad de fabricar por moldeo, componentes libres de defectos de diferentes aleaciones comerciales de magnesio, añadiendo $\mathrm{CaO}$ al caldo, sin emplear $\mathrm{SF}_{6}$. En el caso de las aleaciones $\mathrm{AZ}$ este proceso, además, mejora notablemente las propiedades mecánicas, no sólo por la mayor limpieza de las aleaciones sino también por la presencia de la fase $\mathrm{CaAl}_{2}$ que se forma por la incorporación de calcio al caldo. Este trabajo, enmarcado dentro del proyecto Green Metallurgy (http://www.green-metallurgy.eu) financiado por la Unión Europea (Programa LIFE+2009), estudia la influencia de diferentes adiciones de $\mathrm{CaO}$ en la microestructura y propiedades mecánicas de la aleación Eco-Mg AZ31. El estudio se lleva a cabo en aleaciones AZ31 con 0,5, 1 y 1,5\% CaO procesadas por dos rutas diferentes, aleaciones coladas y extruidas, y aleaciones procesadas por una ruta pulvimetalúrgica utilizando virutas (chips). Esto último tiene como objeto analizar la viabilidad de la reutilización de virutas para la fabricación de componentes de la industria del automóvil y del transporte en general. Se ha demostrado que los materiales fabricados a partir de virutas precompactadas son los más resistentes y la aleación con mayor concentración de $\mathrm{CaO}$ la que presenta los valores más altos de resistencia, cercanos a $320 \mathrm{MPa}$.
\end{abstract}

Palabras clave Eco-Magnesio; AZ31; Microestructura; Propiedades mecánicas.

\section{Mechanical properties of AZ31 alloy processed by a green metallurgy route}

Abstract

Keywords
Recently it has been proved that molding of defect-free components of various commercial alloys of magnesium can be carried out succesfully when small amounts of $\mathrm{CaO}$ are added to the melt, making unnecessary the use of $\mathrm{SF}_{6}$ coverage. In the case of $\mathrm{AZ}$ alloys, this process also remarkably improves their mechanical properties not only by the greater cleaning of alloys but also by the formation of $\mathrm{CaAl}_{2}$ phase. This work, part of the Green project Metallurgy (http://www.green-metallurgy.eu) funded by the European Union (LIFE+2009), studies the influence of different $\mathrm{CaO}$ additions on the microstructure and mechanical properties of AZ31 Eco-Mg alloy. The alloy was processed by a conventional route involving extrusion of as-cast rods as well as by a powder metallurgy route (PM) using chips as starting material. The objective was to analyze the viability of recycling machining chips to manufacture components for the automobile industry and transportation in general, because of its low cost and environmental impact. It has been demostrated that alloys processed from chips exhibit the highest tensile stress values, close to $320 \mathrm{MPa}$.

\section{INTRODUCCIÓN}

Una de las principales razones que ha impulsado la investigación y el desarrollo de aleaciones de magnesio desde finales de la década de los 90 ha sido la creciente concienciación social de la necesidad de ahorrar energía y reciclar. Además, el hecho de que las normativas medioambientales relativas al nivel de las emisiones de gases contaminantes que pueden liberar los medios de transporte sean cada vez más restrictivas, ha motivado que el sector aeronáutico y del automóvil hayan afrontado el reto de disminuir el peso de los componentes, mediante el empleo de materiales más ligeros.

Uno de los problemas derivados del empleo de las aleaciones de magnesio, que limitó su uso hasta los

(•) Trabajo recibido el día 4 de marzo de 2013 y aceptado en su forma final el día 7 de mayo de 2013.

* Politecnico di Milano, Department of Mechanical Engineering, Via La Masa 34, 20156 Milan, Italy.

** Centro Nacional de Investigaciones Metalúrgicas (CENIM-CSIC), Avda. Gregorio del Amo, 8, 28040 Madrid. E-mail: adeva@cenim.csic.es

*** Buhler AG, New Technologies AMT Advanced Materials Division, Gupfenstrasse 5, Uzwil 9240, Switzerland.

*** Casting Research Center, Korea Institute of Industrial Technology (KITECH), 7-47 Songdo-Dong, Yeonsu-gu, Incheon 406-840, Korea. 
años 90, está relacionado con la calidad de los productos colados. Su gran reactividad en estado líquido dificulta la fabricación de aleaciones limpias, exentas de inclusiones. En la actualidad, el hexafluoruro de azufre $\left(\mathrm{SF}_{6}\right)$ es el gas más comúnmente usado como gas de cobertura para evitar la oxidación y/o ignición del caldo durante la fusión y colada. Este gas, sin embargo, es uno de los principales gases que contribuyen al efecto invernadero, de tal modo que el Protocolo de Kyoto ha restringido drásticamente su empleo. Por tanto, la industria del magnesio se ha visto forzada a buscar gases alternativos. Recientemente, S. Kim et al. han demostrado que es factible fabricar componentes de diferentes aleaciones comerciales de magnesio exentos de defectos sin emplear $\mathrm{SF}_{6}$, mediante la adición de $\mathrm{CaO}$ al caldo ${ }^{[1}$ y 2$]$ y las han denominado Eco-Mg (Enviromental Conscious Magnesium). En el caso de las aleaciones AZ, se ha comprobado que este proceso, además, mejora notablemente las propiedades mecánicas de las aleaciones debido a la presencia de partículas de la fase $\mathrm{CaAl}_{2}$ que se forman por la incorporación de calcio al caldo.

En este trabajo se estudia la influencia en la microestructura y en las propiedades mecánicas de diferentes adiciones de $\mathrm{CaO}$ a la aleación $\mathrm{AZ31}$, añadidas durante el proceso de fusión. El estudio se lleva a cabo en aleaciones coladas y extruidas así como en las mismas aleaciones procesadas por una ruta pulvimetalúrgica utilizando virutas de la aleación (chips) precompactadas. El objetivo es demostrar la viabilidad de la reutilización de virutas de mecanizado en el procesado de estas aleaciones para la fabricación de componentes para la industria del automóvil y del transporte en general.

Este estudio está enmarcado dentro del Proyecto Life Green Metallurgy ${ }^{[3]}$ cuyo objetivo es ofrecer una visión práctica del modo de aumentar la eficiencia energética y reducir la huella de carbono (emisión de gases de efecto invernadero) que es demasiado alta en la "no limpia" ruta convencional de fabricación de piezas de magnesio empleada en la actualidad. La viabilidad técnica y económica son puntos claves en el desarrollo de las actividades del citado proyecto, con vistas a conseguir una adopción concreta de las innovaciones del proyecto por parte de grandes sectores de la industria, como por ejemplo, el sector de la automoción. Los resultados que se presentan en este trabajo son los primeros estudios llevados a cabo.

\section{EXPERIMENTAL}

Los materiales estudiados corresponden a la aleación comercial AZ31 modificada con $0,5,1$ y $1,5 \%$ en masa de $\mathrm{CaO}$. Las aleaciones fueron suministradas por Korea Institute of Industrial Technology $(\mathrm{KITECH})$. Los detalles del procedimiento empleado para la fusión y colada de las aleaciones están descritos en la bibliografía ${ }^{[1,2}$ y 4]. La tabla I presenta la composición química de las aleaciones, analizada por absorción atómica. Mediante mecanizado, también realizado en $\mathrm{KITECH}$, se prepararon virutas (chips) de las tres aleaciones.

Las aleaciones coladas en forma de barras de $40 \mathrm{~mm}$ de diámetro se extruyeron a 300 y $350^{\circ} \mathrm{C}$, a una velocidad de extrusión de $2 \mathrm{~mm} / \mathrm{s}$ con una relación de extrusión de 18:1. También se procesó mediante una ruta pulvimetalúrgica esta aleación a partir de los chips. Éstos se compactaron en frío, mediante presión uniaxial, utilizando un útil de $40 \mathrm{~mm}$ de diámetro y $200 \mathrm{~mm}$ de altura y posteriormente se extruyeron utilizando los mismos parámetros que en el caso de las barras coladas.

La caracterización microestructural se llevó a cabo mediante microscopía óptica y electrónica empleando también microanálisis por dispersión de energía. El tamaño de grano se midió mediante el método de intercepción lineal.

Las propiedades mecánicas se estudiaron mediante ensayos de tracción a temperatura ambiente. Se emplearon probetas cilíndricas de $6 \mathrm{~mm}$ de diámetro y $30 \mathrm{~mm}$ de longitud útil. Los materiales se ensayaron a una velocidad inicial de deformación de $10^{-4} \mathrm{~s}^{-1}$. Los resultados de los ensayos son el promedio de al menos tres ensayos.

Tabla I. Composición química de las aleaciones (\% masa)

Table I. Chemical composition of alloys (weight \%)

\begin{tabular}{lccccc}
\hline Aleación & Al & Zn & Ca & Mn & Mg \\
\hline AZ31 + 0,5\% CaO & $3,59 \pm 0,15$ & $0,86 \pm 0,01$ & $0,53 \pm 0,01$ & $0,47 \pm 0,01$ & Resto \\
AZ31 + 1\% CaO & $3,40 \pm 0,15$ & $1,11 \pm 0,01$ & $0,89 \pm 0,01$ & $0,55 \pm 0,01$ & Resto \\
AZ31 + 1,5\% CaO & $3,24 \pm 0,15$ & $0,79 \pm 0,02$ & $1,56 \pm 0,02$ & $0,51 \pm 0,01$ & Resto \\
\hline
\end{tabular}




\section{RESULTADOS Y DISCUSIÓN}

De acuerdo con los estudios realizados por S. Kim et al. ${ }^{[1}$ y 2$]$, las adiciones de $\mathrm{CaO}$ al caldo permiten fundir y colar estas aleaciones de magnesio sin emplear ningún gas protector. Ello es debido a la formación de una capa muy densa y compacta de una mezcla de $\mathrm{MgO}$ y $\mathrm{CaO}$ que protege el caldo de su oxidación continua y por tanto de su inflamación. Este proceso, sin embargo, lleva asociado la incorporación de calcio elemental al líquido, que modifica ligeramente la composición química de la aleación que se manifiesta en cambios microestructurales importantes, especialmente en lo referente a la naturaleza de las fases que solidifican.

\subsection{Microestructura}

La microestructura de colada de las tres aleaciones AZ31 con distintas adiciones de $\mathrm{CaO}$ es similar y está constituida por dendritas de magnesio y diferentes fases en el espacio interdendrítico, que se distinguen por su morfología, tamaño y composición, como se puede ver en la micrografía de electrones retrodispersados de la figura $1 \mathrm{a}$ )-c). Mediante microanálisis por dispersión de energía (Tabla II) se han identificado las siguientes fases: partículas gruesas y brillantes de morfología poligonal cuya composición se acerca a la de la fase $\mathrm{MnAl}$, la fase $\mathrm{CaAl}_{2}$, el eutéctico binario $\mathrm{Mg}+\mathrm{CaAl}_{2}$ y finalmente una fase ternaria CaMgZn. La fracción en volumen de las fases que contienen calcio disminuye con la concentración de dicho elemento en la aleación, de modo que la fase ternaria $\mathrm{CaMgZn}$ aparece muy aisladamente en la aleación con 0,5\% $\mathrm{CaO}$. Es importante resaltar que no se observó en ningún caso la presencia de inclusiones de $\mathrm{MgO}$ ó $\mathrm{CaO}$ procedentes del proceso de colada.
La microestructura de las virutas (chips) obtenidas por mecanizado del material colado es idéntica a la correspondiente al material colado tal y como cabría esperar (Fig. 1 d) - f)). Por tanto, la incorporación de calcio a la aleación AZ31 modifica su microestructura de tal forma que la fase $\mathrm{Mg}_{17} \mathrm{Al}_{12}$, normalmente presente en las aleaciones de la familia AZ, es sustituida por la fases $\mathrm{CaAl}_{2}$ y $\mathrm{CaMgZn}$. Es importante resaltar que la estequiometría de la fase ternaria CaMgZn puede corresponder a la de la fase $\mathrm{Ca}_{2} \mathrm{Mg}_{6} \mathrm{Zn}_{3}{ }^{[5]}$ o bien a la recientemente propuesta por Yi-Nan Zhang ${ }^{[6]}$ con estequiometría $\mathrm{Ca}_{2} \mathrm{Mg}_{\mathrm{x}} \mathrm{Zn}_{15-\mathrm{x}}$ $(4,6 \leq x \leq 12)$. Si se asume que el aluminio está sustituyendo al zinc en esta fase (sus radios atómicos son muy próximos: $\mathrm{r}_{\mathrm{Al}}=1,43 \AA$ y $\mathrm{r}_{\mathrm{Zn}}=1,38 \AA$ ) , los resultados microanalíticos mostrados en la tabla II permiten asignar a esta fase la estequiometría $\mathrm{Ca}_{2} \mathrm{Mg}_{6}(\mathrm{Zn}+\mathrm{Al})_{3}$.

Tras el proceso de extrusión, los dos materiales, el colado y el procesado mediante chips, presentan una microestructura con las diferentes fases fragmentadas y alineadas en la dirección de extrusión (Fig. 2). En la figura 3 se presenta la imagen de electrones retrodispersados de cada una de las fases presentes así como su composición obtenida mediante microanálisis en el caso de los materiales con 1,5\% CaO extruidos a $300^{\circ} \mathrm{C}$. Es preciso resaltar que la composición de las fases $\mathrm{CaAl}_{2}$ y de la ternaria CaMgZn son algo diferentes a las analizadas en las aleaciones coladas. En esta última se observa un pequeño aumento del contenido de aluminio con respecto al medido en esta fase en las aleaciones coladas, así como un ligero cambio en su morfología (Fig. 1 b)) y Fig. 3). Además, esta fase no está presente en los materiales extruidos a $350^{\circ} \mathrm{C}$. Estos resultados están de acuerdo con el estudio de Levi et al. ${ }^{[5]}$ en aleaciones ternarias $\mathrm{Mg}-\mathrm{Ca}-\mathrm{Zn}$ en el que concluyen que la fase $\mathrm{Ca}_{2} \mathrm{Mg}_{6} \mathrm{Zn}_{3}$ es estable hasta aproximadamente $350^{\circ} \mathrm{C}$, reforzando la idea de que la fase ternaria que

Tabla II. Composición de las diferentes fases presentes en la

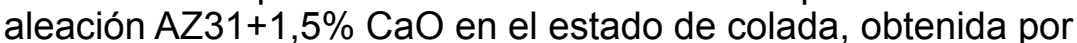
microanálisis (at.\%)

Table II. Chemical composition (\%at.) of different phases present in the as-cast $A Z 31+1.5 \% \mathrm{CaO}$ alloy

\begin{tabular}{lccrrr}
\hline $\begin{array}{l}\text { Composición } \\
\text { (at.\%) }\end{array}$ & Al & Ca & Mn & Zn & Mg \\
\hline Fase CaMgZn & 8,6 & 15,8 & 0,1 & 20,1 & resto \\
Fase CaAl 2 & 52 & 27 & 0,1 & 1,7 & resto \\
Fase MnAl & 48,1 & 0,4 & 47,8 & 0,1 & resto \\
Matriz & 1,1 & 0,2 & 0,1 & 0,6 & resto \\
\hline
\end{tabular}



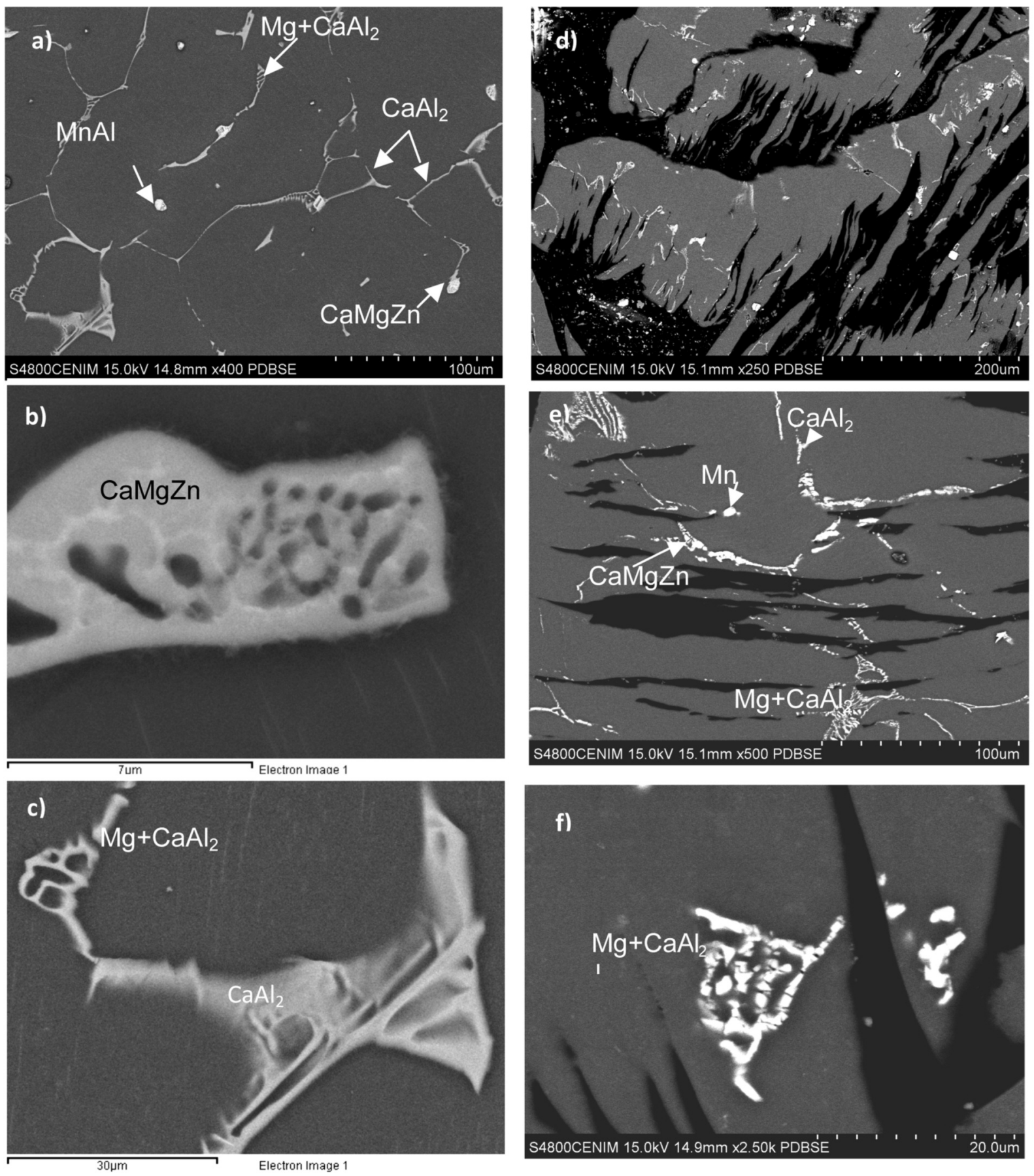

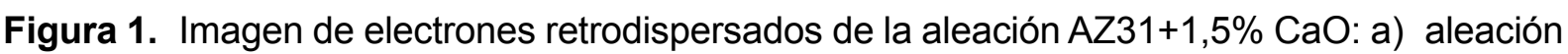
colada; b) y c) detalle de las fases de la aleación colada; d) viruta; e) y f) detalle de las segundas fases en la viruta.

Figure1. Backscattered electron image of AZ31+1.5\% CaO alloy: a) cast alloy; b) and c) detail of the second phases; d) chip; e) and f) detail of second phases.

solidifica en la aleación AZ31 modificada con $\mathrm{CaO}$ es la fase $\mathrm{Ca}_{2} \mathrm{Mg}_{6}(\mathrm{Zn}+\mathrm{Al})_{3}$. Además, el cambio de morfología observado en la fase ternaria tras la extrusión a $300^{\circ} \mathrm{C}$ está relacionado probablemente con el inicio de su disolución.
El tamaño de grano de los dos materiales extruidos es bimodal caracterizado por áreas de grano grueso y otras de grano muy fino completamente recristalizado, tal y como se comprueba en las micrografías ópticas de la figura 4. Con el fin de establecer la influencia 

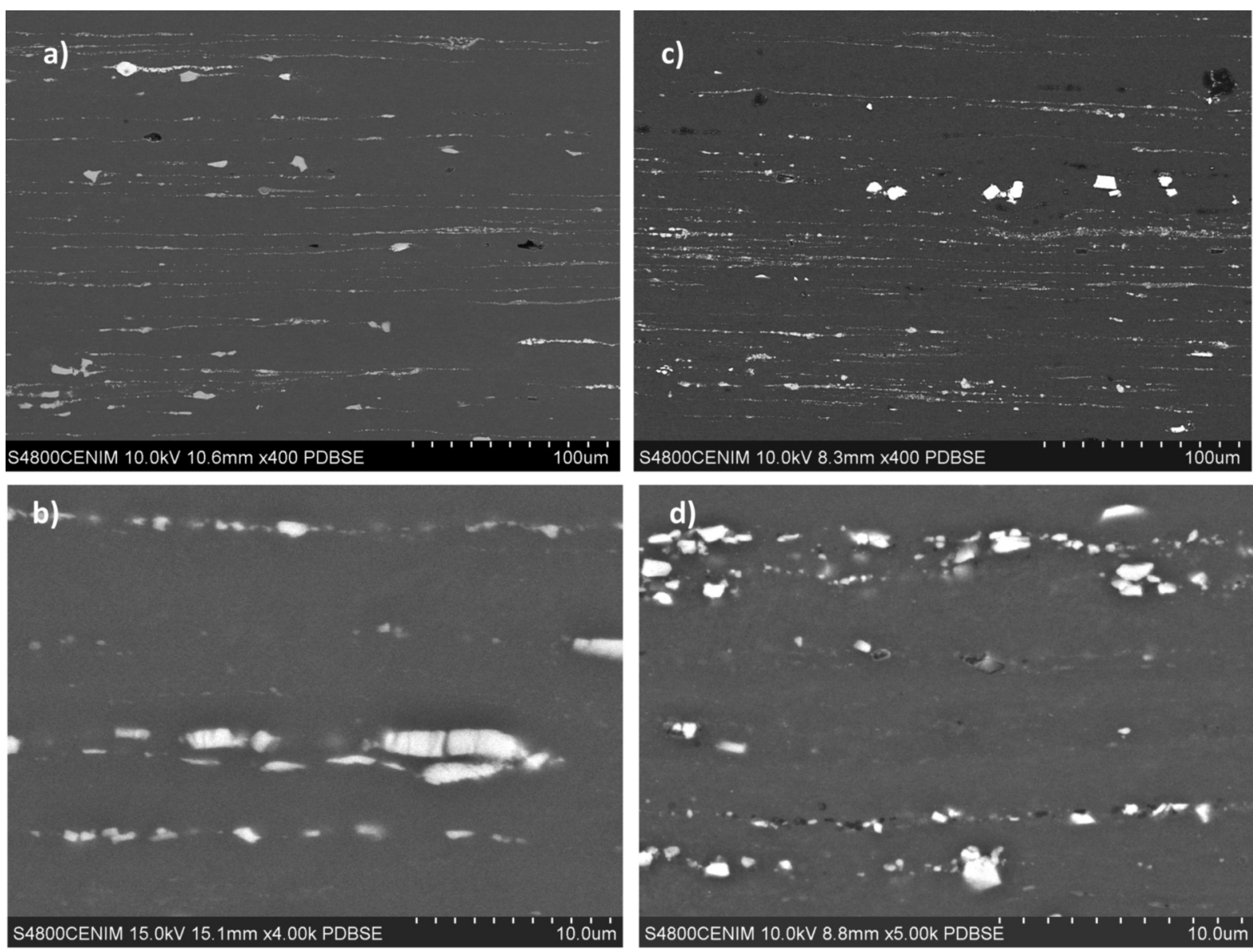

Figura 2. Imagen de electrones retrodispersados de la aleación $\mathrm{AZ31}+1,5 \% \mathrm{CaO}$ : a ) y b) aleación colada y extruida a $300^{\circ} \mathrm{C}$; c) y d) virutas precompactadas y extruidas a $300^{\circ} \mathrm{C}$.

Figure 2. Backscattered electron image of $A Z 31+1.5 \% \mathrm{CaO}$ alloy: a) and b) cast and extruded at $300^{\circ} \mathrm{C}$; c) and d) chips extruded at $300^{\circ} \mathrm{C}$.

Tabla III. Tamaño de grano de los materiales extruidos medido por el método de intercepción lineal

Table III. Grain size of extruded materials determined by lineal interception method

\begin{tabular}{|c|c|c|c|}
\hline Aleación & Material & T. extru. $\left({ }^{\circ} \mathrm{C}\right)$ & $\begin{array}{l}\text { Tamaño de } \\
\text { grano }(\mu \mathrm{m})\end{array}$ \\
\hline \multirow{2}{*}{$\mathrm{AZ} 31+0,5 \% \mathrm{CaO}$} & Colado & $\begin{array}{l}300 \\
350\end{array}$ & $\begin{array}{r}8 \pm 0,7 \\
18 \pm 1,0\end{array}$ \\
\hline & $\begin{array}{c}\text { Virutas } \\
\text { precompactadas }\end{array}$ & $\begin{array}{l}300 \\
350\end{array}$ & $\begin{array}{l}2,9 \pm 0,1 \\
5,6 \pm 0,2\end{array}$ \\
\hline \multirow{2}{*}{$\mathrm{AZ31}+1,0 \% \mathrm{CaO}$} & Colado & $\begin{array}{l}300 \\
350\end{array}$ & $\begin{array}{r}9 \pm 0,5 \\
16 \pm 1,7\end{array}$ \\
\hline & $\begin{array}{c}\text { Virutas } \\
\text { precompactadas } \\
\end{array}$ & $\begin{array}{l}300 \\
350 \\
\end{array}$ & $\begin{array}{l}3,3 \pm 0,1 \\
5,5 \pm 0,1\end{array}$ \\
\hline \multirow{2}{*}{$\mathrm{AZ} 31+1,5 \% \mathrm{CaO}$} & Colado & $\begin{array}{l}300 \\
350\end{array}$ & $\begin{array}{r}2,5 \pm 0,8 \\
6 \pm 0,3 \\
\end{array}$ \\
\hline & $\begin{array}{c}\text { Virutas } \\
\text { precompactadas }\end{array}$ & $\begin{array}{l}300 \\
350\end{array}$ & $\begin{array}{r}3,2 \pm 0,2 \\
5 \pm 0,3\end{array}$ \\
\hline
\end{tabular}




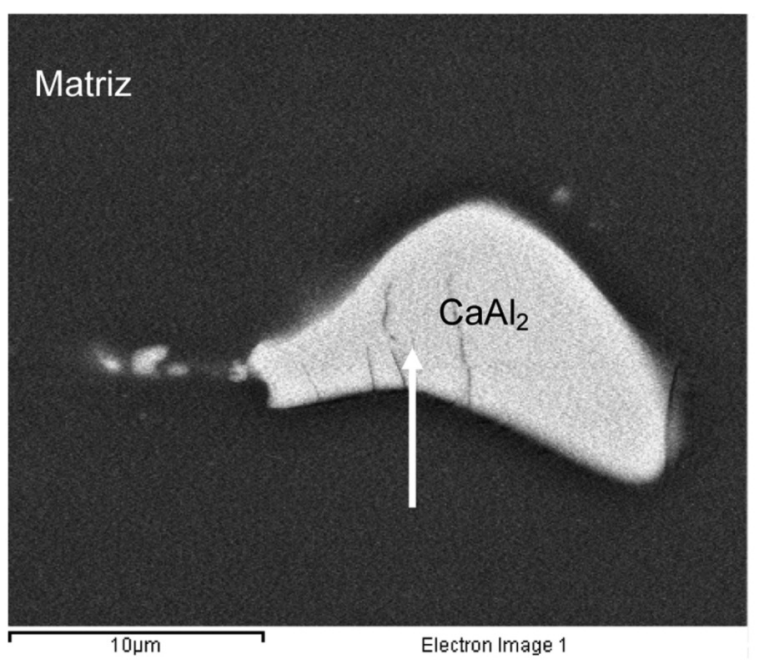

Electron Image 1

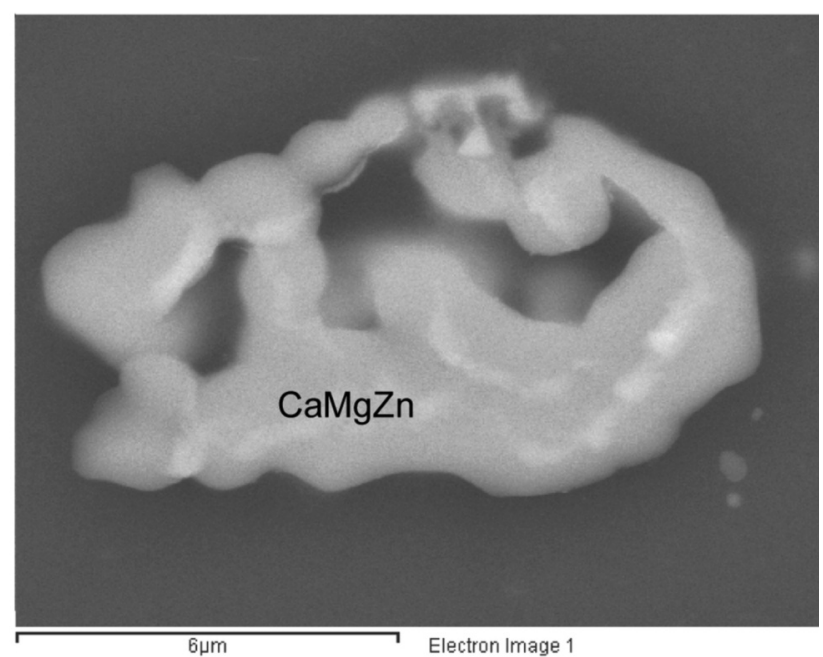

Electron Image 1

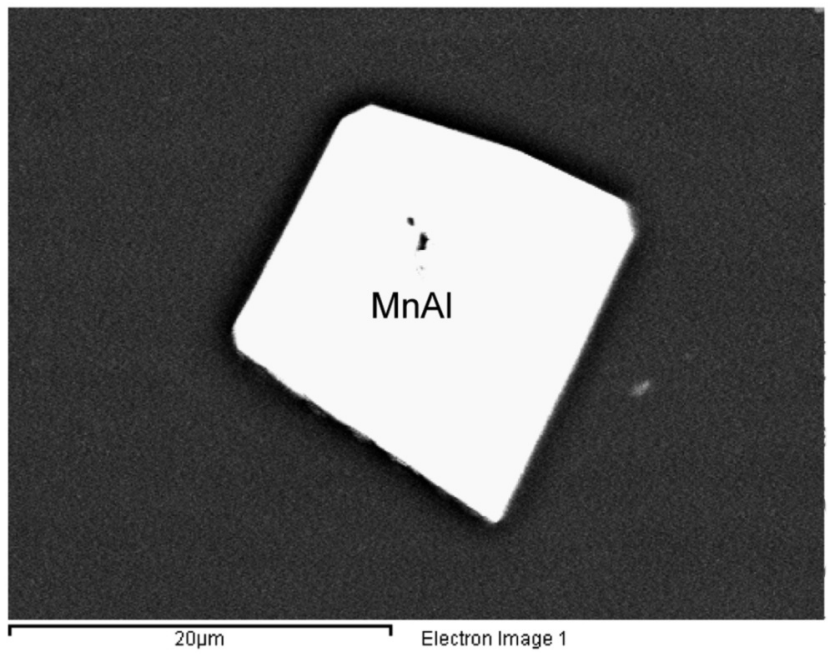

\begin{tabular}{lrrrrr}
\hline $\begin{array}{l}\text { Comp. } \\
\text { \% at. }\end{array}$ & Mg & Al & Ca & Mn & Zn \\
\hline $\mathrm{CaAl}_{2}$ & 8,9 & 60,9 & 28,2 & 0,05 & 1,9 \\
$\mathrm{CaMgZn}$ & 49,6 & 15,3 & 13,2 & 0,1 & 21,9 \\
$\mathrm{MnAl}$ & 0,9 & 51,5 & 0,1 & 47,4 & 0,2 \\
Matriz & 97,9 & 1,5 & 0,3 & 0,1 & 0,2 \\
\hline
\end{tabular}

Figura 3. Detalle de las diferentes fases que aparecen en los dos materiales extruidos de la

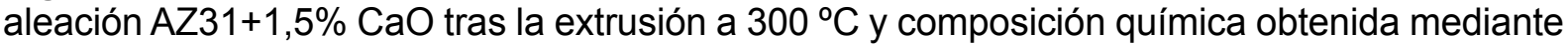
microanálisis.

Figure 3. Detail of different phases present in both AZ31+1.5\% Ca O alloys after extrusion at $300^{\circ} \mathrm{C}$ and composition obtained by microanalysis.

del contenido de $\mathrm{CaO}$ en la microestructura de las aleaciones, se calculó el tamaño promedio de grano por el método de intercepción lineal. Los resultados, listados en la tabla.III, indican que la temperatura de extrusión ejerce una gran influencia en el tamaño de grano cuando el $\mathrm{CaO}$ se añade en bajas concentraciones. Así en el caso de las aleaciones con 0,5 y $1 \% \mathrm{CaO}$, el tamaño de grano de las aleaciones extruidas a $350^{\circ} \mathrm{C}$ es el doble del de los materiales extruidos a $300^{\circ} \mathrm{C}$. Además, contenidos crecientes de $\mathrm{CaO}$ producen un refinamiento microestructural importante en el caso de las aleaciones coladas y extruidas disminuyendo desde $18 \mu \mathrm{m}$, para la aleación con 0,5\% CaO extruida a a $350^{\circ} \mathrm{C}$, hasta $6 \mu \mathrm{m}$ en el caso de la aleación con 1,5\% Ca. Estas observaciones y resultados están rela- cionados directamente con la fracción en volumen de segundas fases presentes en las aleaciones coladas.

El material procesado con virutas es el que presenta microestructuras con tamaño de grano más fino y menos dependiente del contenido en $\mathrm{CaO}$.

\subsection{Propiedades mecánicas}

Las curvas tensión-deformación de los dos materiales extruidos a 300 y $350^{\circ} \mathrm{C}$ se muestran en la figura 5 . Las curvas indican que los materiales fabricados con chips precompactados, son los que alcanzan valores más altos de resistencia mecánica. 
PROPIEDADES MECÁNICAS DE LA ALEACIÓN AZ31 PROCESADA POR UNA RUTA ECO-SOSTENIBLE MECHANICAL PROPERTIES OF AZ31 ALLOY PROCESSED BY A GREEN METALLURGY ROUTE
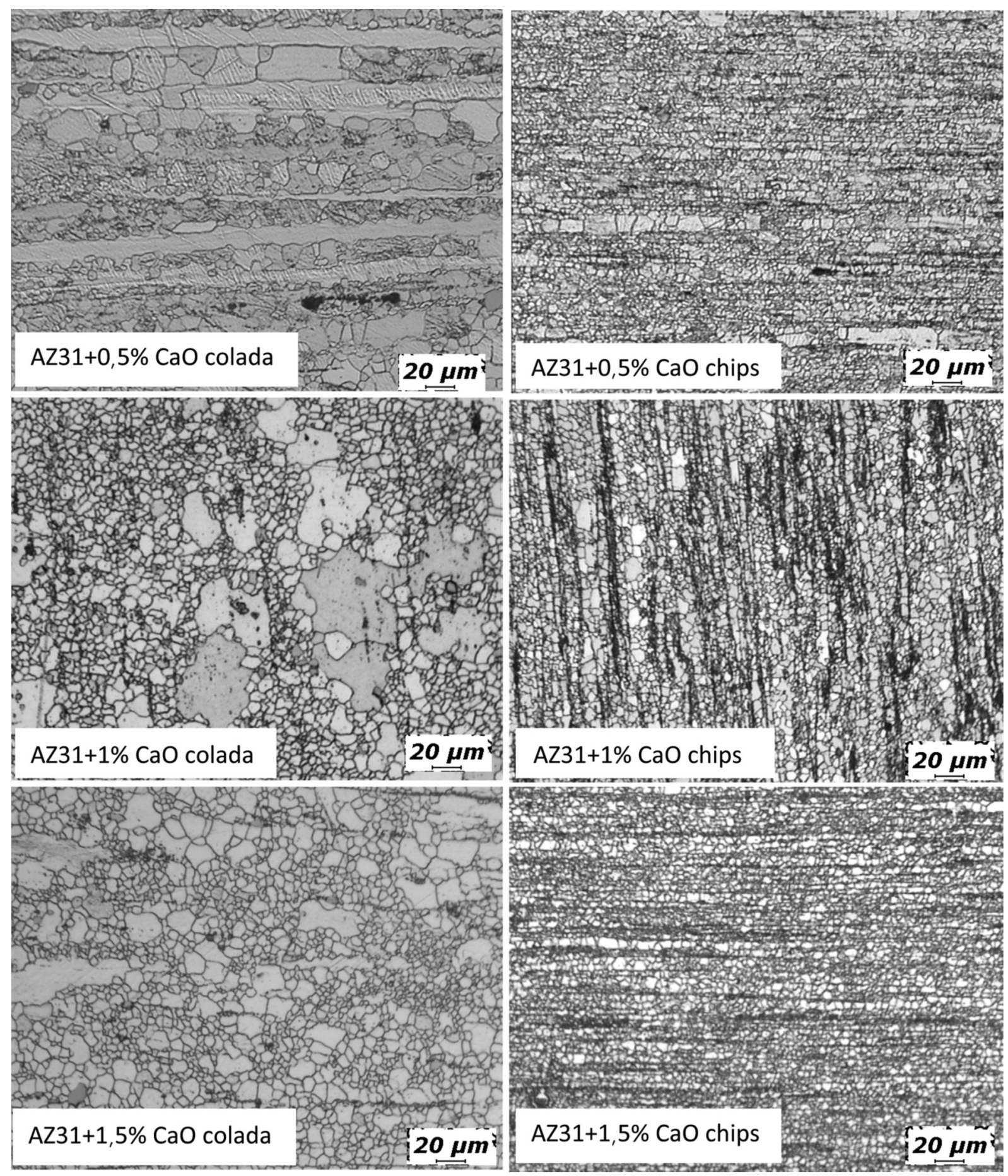

Figura 4. Sección metalográfica longitudinal de los materiales extruidos a $350^{\circ} \mathrm{C}$ mostrando la estructura de granos.

Figure 4. Longitudinal section of extruded bars at $350^{\circ} \mathrm{C}$ showing the grain structure.

En la tabla IV se presentan los valores medios de límite elástico, resistencia a la tracción y alargamiento a rotura de los materiales estudiados. De esta tabla se deduce que comparando entre sí materiales con los mismos contenidos en $\mathrm{CaO}$ y la misma ruta de procesado, los extruidos a $300^{\circ} \mathrm{C}$ son los que presen- tan valores más altos de límite elástico y resistencia mecánica lo que puede atribuirse a su menor tamaño de grano. También se observa una clara dependencia del contenido en $\mathrm{CaO}$, o fracción en volumen de segundas fases, en el límite elástico. Así, para la misma temperatura de extrusión y ruta de procesado, los 

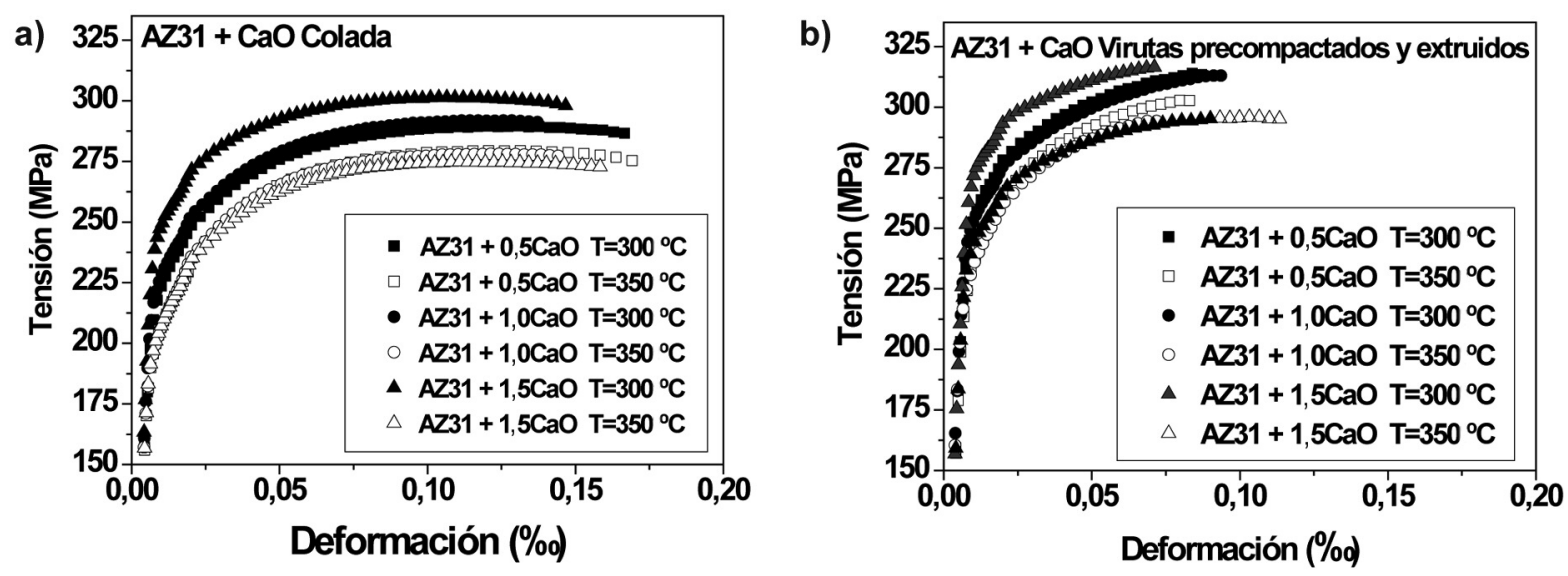

Figura 5. Curvas tensión-deformación de las aleaciones extruidas a 300 y $350^{\circ} \mathrm{C}$ : a) AZ31+CaO

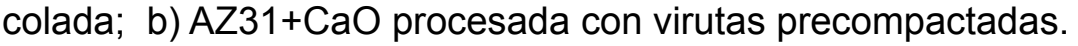

Figure 5. Tensile stress-strain curves of extruded alloys at 300 and $350^{\circ} \mathrm{C}$ : a) AZ31+CaO cast alloy; b) $A Z 31+C a O$ processed with chips.

materiales con concentraciones del $1,5 \% \mathrm{CaO}$ son los que poseen mayores valores de este parámetro. Respecto al efecto del procesado, se comprueba que los materiales fabricados a partir de chips precompatados son más resistentes. Entre ellos, la aleación con mayor concentración de $\mathrm{CaO}$ es la que presenta los valores más altos de resistencia, cercanos a $320 \mathrm{MPa}$, lo cual está íntimamente relacionado con el tamaño de grano más fino.
En cuanto al alargamiento a rotura no se encuentra dependencia ni con la temperatura de extrusión ni con la concentración de CaO. No obstante, sí existe una disminución notable vinculada a la ruta de procesado. Así, el material fabricado a partir de chips alarga aproximadamente un $9 \%$ en promedio, la mitad que el material colado y extruido. No obstante, las superficies de fractura de todos los materiales indican que se tratan de roturas dúctiles por

Tabla IV. Valores promedio de las propiedades mecánicas en tracción de las aleaciones en función del proceso de fabricación y de la temperatura de extrusión

Table IV. Average values of tensile mechanical properties of materials as a function of the processing route and extrusion temperature

\begin{tabular}{|c|c|c|c|c|c|}
\hline Material & Proceso & $\begin{array}{l}\text { T. extru. } \\
\left({ }^{\circ} \mathrm{C}\right)\end{array}$ & $\begin{array}{l}\text { LE } \\
\text { (MP) }\end{array}$ & $\begin{array}{c}\mathrm{Rm} \\
(\mathrm{MPa})\end{array}$ & $\varepsilon(\%)$ \\
\hline \multirow{2}{*}{ 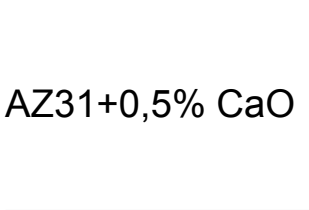 } & Colada & $\begin{array}{l}300 \\
350\end{array}$ & $\begin{array}{l}201,8 \\
187,3\end{array}$ & $\begin{array}{l}289,3 \\
279\end{array}$ & $\begin{array}{l}16,3 \\
18,1\end{array}$ \\
\hline & $\begin{array}{c}\text { Virutas } \\
\text { precompactadas }\end{array}$ & $\begin{array}{l}300 \\
350\end{array}$ & $\begin{array}{l}230,8 \\
219,3\end{array}$ & $\begin{array}{l}313,5 \\
304,5\end{array}$ & $\begin{array}{l}9,2 \\
8,3\end{array}$ \\
\hline \multirow{2}{*}{$\mathrm{AZ} 31+1,0 \% \mathrm{CaO}$} & Colada & $\begin{array}{l}300 \\
350\end{array}$ & $\begin{array}{l}209 \\
191,5\end{array}$ & $\begin{array}{l}291 \\
277,8\end{array}$ & $\begin{array}{l}14,8 \\
16,9\end{array}$ \\
\hline & $\begin{array}{c}\text { Virutas } \\
\text { precompactadas }\end{array}$ & $\begin{array}{l}300 \\
350\end{array}$ & $\begin{array}{l}236,3 \\
212\end{array}$ & $\begin{array}{l}312,3 \\
291,5\end{array}$ & $\begin{array}{l}9,2 \\
7,2\end{array}$ \\
\hline \multirow{2}{*}{ 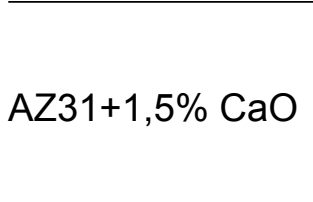 } & Colada & $\begin{array}{l}300 \\
350\end{array}$ & $\begin{array}{l}230,8 \\
193\end{array}$ & $\begin{array}{l}301,8 \\
276,3\end{array}$ & $\begin{array}{l}15,5 \\
16,6\end{array}$ \\
\hline & $\begin{array}{c}\text { Virutas } \\
\text { precompactadas }\end{array}$ & $\begin{array}{l}300 \\
350\end{array}$ & $\begin{array}{l}251,5 \\
223,3\end{array}$ & $\begin{array}{l}317,8 \\
292,3\end{array}$ & $\begin{array}{r}9 \\
10\end{array}$ \\
\hline
\end{tabular}


PROPIEDADES MECÁNICAS DE LA ALEACIÓN AZ31 PROCESADA POR UNA RUTA ECO-SOSTENIBLE MECHANICAL PROPERTIES OF AZ31 ALLOY PROCESSED BY A GREEN METALLURGY ROUTE

crecimiento y coalescencia de microcúpulas nucleadas en las partículas de las fases $\mathrm{CaAl}_{2}$ y $\mathrm{MnAl}$. En la figura 6 se muestran las superficies de fractura de la aleación $\mathrm{AZ31}+1,5 \% \mathrm{CaO}$ extruida a $350^{\circ} \mathrm{C}$. En el caso de la aleación colada se observa que las microcúpulas son el resultado de la rotura de la segunda fase, como se puede apreciar en la micrografía de electrones retrodispersados de la figura 6 b). En el caso del material fabricado a partir de virutas parece que la microcúpula se genera por despegue de la intercara fase/matriz. Además, la fractura en ambos materiales es de tipo intergranular y el menor tamaño de grano del material procesado con virutas explica la menor plasticidad de este material con respecto al colado.

Para evaluar la influencia en las propiedades mecánicas de la incorporación de calcio en la composición de la aleación AZ31 así como el empleo de chips para su fabricación, se comparan en los dia- gramas de barras de la figura 7 las propiedades en tracción de la aleación $\mathrm{AZ31}+1,5 \% \mathrm{CaO}$ con otras de la bibliografía correspondientes a aleaciones comerciales y aleaciones PM fabricadas a partir de polvos solidificados rápidamente. Se observa que las propiedades en tracción de la aleación procesada en este trabajo mediante chips, son comparables a las de la aleación AZ31 PM procesada con polvos solidificados rápidamente de tamaño de grano de $5 \mu \mathrm{m}$, y superiores a las de la aleación comercial colada y extruida.

Como se ha comentado en la Introducción, estos resultados son los preliminares de un estudio más amplio que se está llevando a cabo dentro del Proyecto Life. En la actualidad se está analizando la influencia de las segundas fases en las propiedades mecánicas así como la disolución de la fase ternaria, en el caso de los materiales extruidos a $350^{\circ} \mathrm{C}$.
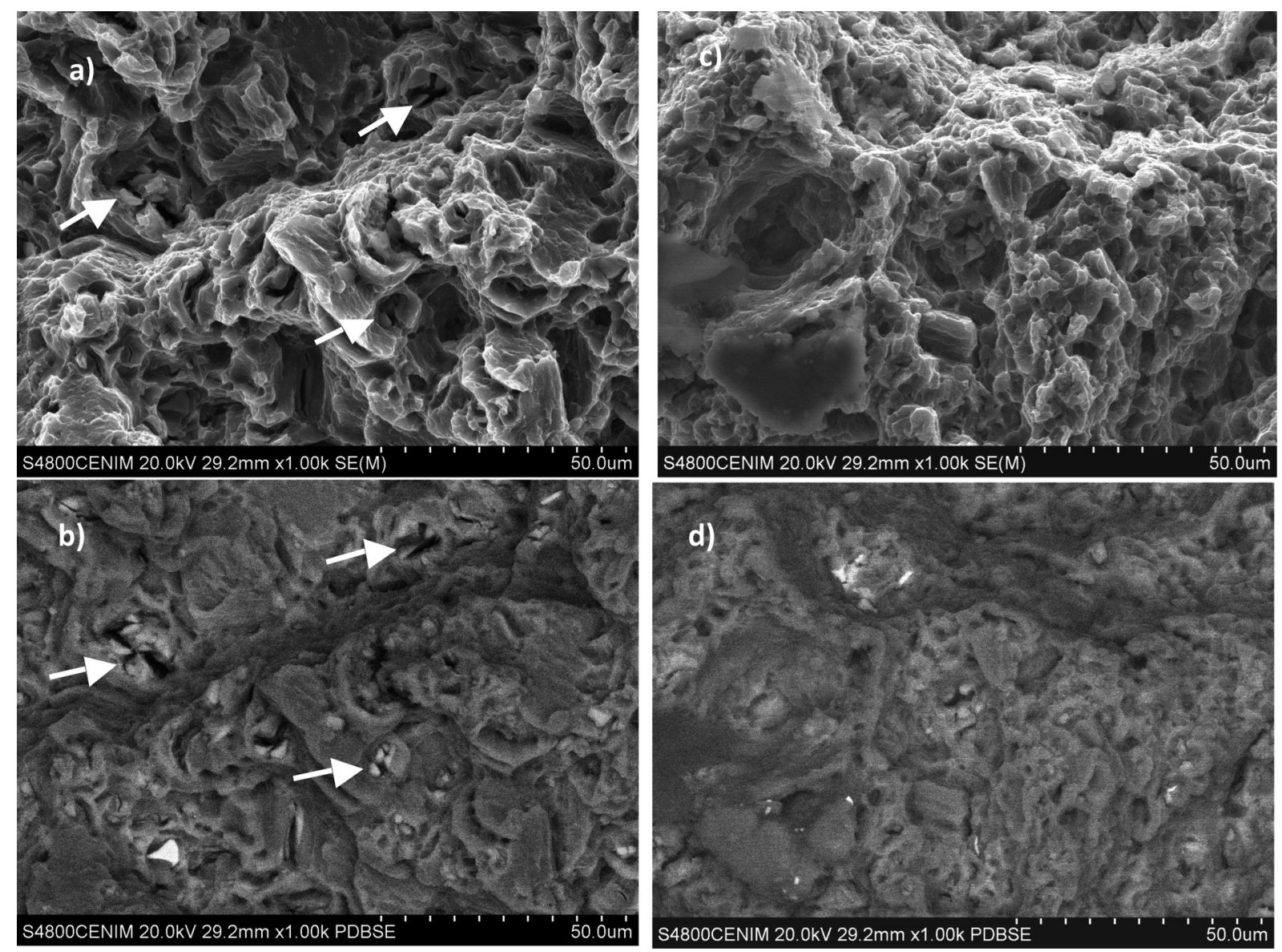

Figura 6. Superficie de fractura de la aleación $\mathrm{AZ31}+1,5 \% \mathrm{CaO}$ extruida a $350^{\circ} \mathrm{C}$ y ensayada en tracción a temperatura ambiente: a) y b) aleación colada; c) y d) aleación procesada con virutas precompactadas. Las flechas señalan partículas de segunda fase rotas.

Figure 6. Fracture surface of tensile tested $\mathrm{AZ31}+1.5 \% \mathrm{CaO}$ alloy extruded at $350^{\circ} \mathrm{C}$ : a) and b) as-cast alloy; c) and d) precompacted chips. Arrows show second-phases particles broken. 


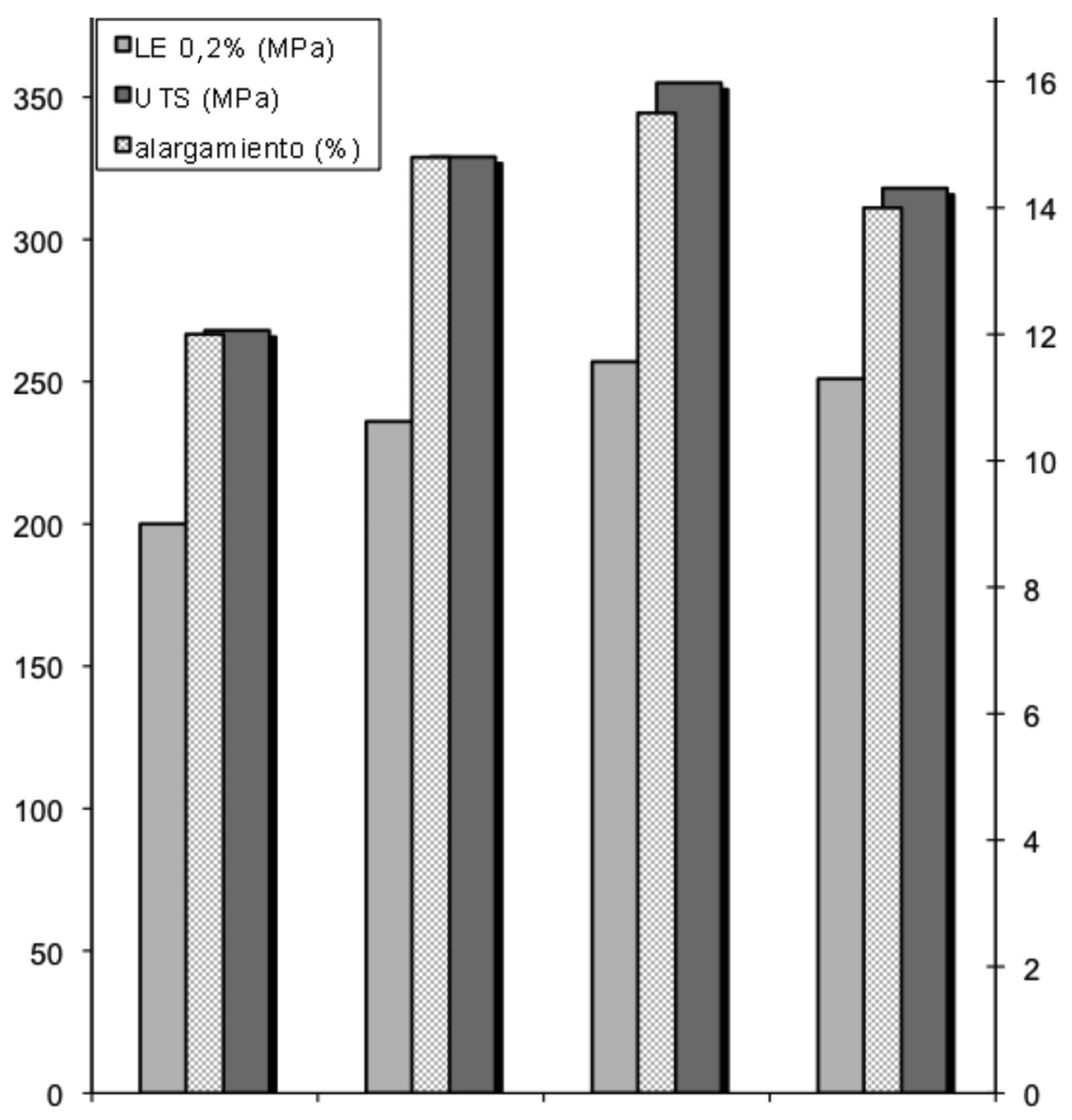

Figura 7. Comparación de las propiedades mecánicas de la aleación $\mathrm{AZ} 31+1,5 \% \mathrm{CaO}$ procesada con virutas y extruida a $300^{\circ} \mathrm{C}$ con datos tomados de la bibliografía ${ }^{77}$ y 8$]$.

Figure 7. Mechanical properties of $A Z 31+1.5 \% \mathrm{CaO}$ alloy processed from chips compared with bibliography data ${ }^{[7}$ and 8$]$.

\section{CONCLUSIONES}

- Este estudio ha demostrado la posibilidad de fabricar la aleación Eco-AZ31 por colada, exenta de óxidos. sin emplear gases protectores, mediante la adición de $\mathrm{CaO}$.

- La incorporación del calcio a la aleación modifica la naturaleza de las fases que solidifican, reemplazándose la fase $\mathrm{Mg}_{17} \mathrm{Al}_{12}$ por la fase $\mathrm{CaAl}_{2}$ y la fase ternaria $\mathrm{Ca}_{2} \mathrm{Mg}_{6}(\mathrm{Zn}+\mathrm{Al})_{3}$. Esta fase que aparece tanto en la estructura de colada como en los materiales extruidos a $300^{\circ} \mathrm{C}$, se disuelve tras la extrusión a $350^{\circ} \mathrm{C}$.

- Concentraciones crecientes de $\mathrm{CaO}$ aumentan el límite elástico y la resistencia mecánica de la aleación AZ31, siendo la fractura siempre de tipo dúctil.

- Se ha comprobado que la aleación procesada por una ruta pulvimetalúrgica, empleando virutas de mecanizado, tiene propiedades mecánicas com- parables a las de la aleación AZ31 fabricada a partir de polvos solidificados rápidamente y superiores a las de la aleación comercial AZ31 extruida.

\section{Agradecimientos}

Este trabajo ha sido financiado por la Unión Europea (Programa LIFE09 ENV IT 117 “Green Metallurgy”).

\section{REFERENCIAS}

[1] J.K. Lee y S.K. Kim, Trans. Nonferrous Metals Society of China 21, 1 (2011) 23-27.

[2] S.K. Kim, J.K. Lee, Y. Yoon y H. Jo, J. Mater. Process. Tech. 187-188 (2007) 757-760.

[3] (http://www.green-metallurgy.eu): (C) 2011 Green Metallurgy Innovation. 
[4] J.K. Lee, H. Jo y S.K. Kim, Rare Metals 25 (2006) 155-159.

[5] G. Levi, S. Avraham, A. Zilverov y M. Bamberger, Acta Mater. 54 (2006) 521-530.

[6] Y. Nan Zhang, D. Kervokov, L. Jian, E. Essadiqui y M. Medraj, Intermetallics 18 (2010) 2.404-2.411.
[7] Magnesium and Magnesium alloys, ASM Specialty Handbook, Ed. M.M. Avedesian y H. Baker, ASM International, 1999, p. 172.

[8] S. Cabeza Sánchez, Máster Oficial en Materiales Estructurales para las Nuevas Tecnologías, URJC y UC3M, Junio, 2010. 